\title{
Factores determinantes de la satisfacción del paciente en tratamiento renal sustitutivo
}

Premio Bellco de Investigación en Técnicas de Diálisis

Hernández Meca, Ma E ncarnación* - Ochando García, Antonio* - Lorenzo Martínez, Susana **

Orbes Cervantes, Pablo** - López Revuelta, Katia*

*Unidad de Diálisis - **Unidad de Calidad - Fundación Hospital Alcorcón

\section{Resumen}

El interés de los profesionales de enfermería se ha centrado siempre en prestar cuidados de calidad, sería errónea esta concepción sin tener en cuenta la opinión de los pacientes. Los objetivos del estudio son: 1. Determinar el grado de satisfacción y capacidad funcional de los pacientes en diálisis, valorando si existe diferencia según el tratamiento sustitutivo que realicen. 2 . I dentificar los aspectos del proceso asistencial que generan mayor satisfacción.

E studio descriptivo transversal a todos los pacientes de la unidad en marzo-abril 2005. Se administró el cuestionario SERVQHOS modificado. Se utilizó el índice de Karnosky para medir el grado de autonomía.

Para el análisis de datos se utilizó el programa SPSS obteniendo las frecuencias y medias de puntación en cada ítem. Se utilizó el Alpha de Cronbach para el análisis de fiabilidad. Mediante análisis factorial se identificaron los atributos determinantes de la satiffacción.

\section{Correspondencia:}

M a Encarnación Hernández M eca

C/ Rozabellas no 11

28230 Las Rozas (M adrid)

e-mail: mehernandez@fhalcorcon.es
Se analizaron 77 cuestionarios. Tasa de respuesta del $95 \%$, Las características demográficas son las siguientes: de los pacientes encuestados HD/DP $55 / 22$, el $65 / 85 \%$ de los pacientes eran hombres. E dad media $68,8 \pm 14 / 54,6 \pm 12,4$ años. El alpha de Cronbach fue de 0,95/0,90. EI grado de satisfacción de los pacientes de HD es 3,28, en los de DP 3,68.

No se ha encontrado correlación entre la capacidad funcional y la satisfacción de los pacientes en diálisis. Las variables que determinan el grado de satisfacción de los pacientes en HD son aquellas relacionadas con la atención que reciben. E n DP están relacionadas con la responsabilidad de asumir la técnica.

Palabras clave:

SATISFACCIÓN DE PACIENTES

CALIDAD PERCIBIDA

CAPACIDAD FUNCIONAL

DIÁLISIS

Factors determining patient satisfaction in kidney replacement treatment

\section{Abstract}

The interest of nursing professionals has always been focused on providing quality care. This focus would be incorrect if it failed to take into account patients' opinions. The objectives of the study are: 
1. To determine the degree of satisfaction and functional capacity of patients on dialysis, evaluating whether there is a difference depending on the replacement treatment they receive, and 2 . To identify the aspects of the care process that generate greatest satisfaction.

Transversal descriptive study of all the patients in the unit in March-April 2005. The modified SERVQH OS questionnaire was administered. The Karnofsky index was used to measure the degree of autonomy.

To analyse the data the SPSS program was used, obtaining score frequencies and averages on each item. Cronbach's Alpha was used to analyse reliability. The attributes that determined satisfaction were identified using factorial analysis.

A total of 77 questionnaires were analysed. Response rate of $95 \%$. The demographic characteristics are as follows: of the patients surveyed HD/PD 55/22, 65/85\% of the patients were men. Average age $68.8 \pm 14 / 54.6 \pm 12.4$ years. Cronbach's Alpha was $0.95 / 0.90$. The degree of satisfaction is 3.28 in HD patients, and 3.68 in PD patients.

No correlation was found between functional capacity and the satisfaction of patients on dialysis. The variables that determine the degree of satisfaction of patients on HD are those related to the attention they receive. In DP they are related to the responsibility of undertaking the technique.

\section{KEY WORDS: \\ PATIENT SATISFACTION \\ PERCEIVED QUALITY \\ FUNCTIONAL CAPACITY \\ DIALYSIS}

\section{Introducción}

El concepto de salud y atención sanitaria se ha ido actualizando e impregnando de valores según el momento histórico y social. A lo largo de las últimas décadas se concibe el concepto de salud positiva, como algo más que la ausencia de enfermedad o discapacidad y varía su percepción en función de que preguntemos al paciente, a la familia, al médico, etc ${ }^{1,2,3}$.

E $n$ el actual sistema sanitario, el ciudadano ha pasado a ser el centro del mismo. Para los profesionales sanitarios y los pacientes es importante que las atenciones que dispensan los primeros sean efectivas (ejerzan un efecto positivo en los niveles de salud de los segundos), eficientes (ese efecto debería lograrse a un coste asumible y, por ejemplo, no impedir el tratamiento de otros pacientes), aceptables (para el paciente que lo recibe y en opinión del conjunto de la profesión), accesibles (para el paciente en términos de distancia, esperas, costes, etc.), han de ser valoradas como útiles por los propios pacientes (por ejemplo en términos de calidad de vida experimentada a partir de la aplicación de una terapia determinada) y deben estar basadas en evidencias (decisiones tomadas en base al conocimiento empírico y no en intuiciones) $)^{4,5}$.

La práctica asistencial del personal de enfermería comprende la valoración, el diagnóstico y el tratamiento de la respuesta humana a los problemas percibidos, reales 0 potenciales, físicos 0 psicosociales que pueden ser episódicos, primarios y/o agudos en el cuidado de los pacientes ${ }^{6}$. E I interés de los profesionales de enfermería se ha centrado siempre en prestar cuidados sanitarios de calidad, sin embargo sería errónea esta concepción sin tener en cuenta la opinión de los pacientes. E s por ello que en los últimos años han cobrado importancia aspectos tales como la calidad de vida, salud percibida y satisfacción del enfermo, a pesar de ello aún conocemos poco de los factores que influyen en los mismos ${ }^{7,8}$.

Son muchos y variados los aspectos que deben abordarse al referirnos al paciente renal. Sobre todo si tenemos en cuenta el aumento de la incidencia y prevalencia ${ }^{9}$ de la insuficiencia renal crónica, el incremento del coste del tratamiento renal sustitutivo de los pacientes con esta patología ${ }^{10}$, así como el cambio en el perfil de los pacientes ${ }^{11}$.

Respecto a los resultados percibidos por el paciente, tales como la calidad de vida relacionada con la salud, los pacientes en diálisis en algunos estudios muestran peores puntuaciones que la población ge- 
neral, y se observa como la capacidad funcional del paciente se relaciona con la calidad de vida relacionada con la salud ${ }^{12}$.

\section{Objetivo}

E I objetivo principal de este estudio ha sido determinar el grado de satisfacción de los pacientes en diálisis con relación a la atención recibida para identificar oportunidades de mejora.

\section{Objetivos específicos:}

- Determinar la capacidad funcional de los pacientes en hemodiálisis y diálisis peritoneal de la unidad.

- Valorar si existen diferencias en el nivel de satisfacción según el tipo de tratamiento renal sustitutivo que recibe el paciente y/o la capacidad funcional que presentan.

- Conocer los aspectos del proceso asistencial que determinan la satisfacción de los pacientes.

\section{Material y método}

Estudio observacional descriptivo transversal a los pacientes en tratamiento renal sustitutivo con hemodiálisis (HD) y diálisis peritoneal (DP) de la F undación Hospital Alcorcón durante los meses de febrero y marzo de 2005. De los 89 pacientes en tratamiento, uno fue excluido por deterioro cognitivo severo, dos pacientes declinaron participar y dos pacientes en tratamiento domiciliario con DP no acudieron al centro a ninguna revisión durante el periodo de estudio. Tras informar de los objetivos del estudio y solicitar su consentimiento verbalmente, se entregaron un total de 84 cuestionarios. Tres pacientes fallecieron antes de cumplimentar dicho cuestionario.

Instrumento de evaluación. E I personal de Enfermería de la U nidad de Diálisis distribuyó un cuestionario, facilitando la información e instrucciones necesarias para que cada paciente lo cumplimentara en su domicilio de forma anónima y lo depositara en un buzón colocado al efecto en la unidad. Aquellos pacientes analfabetos o que presentaban déficits sensoriales para cumplimentar el cuestionario fueron ayudados por sus familiares. Al momento de entrega de dicho cuestionario dos enfermeros -que habían demostrado concordancia en la valoración- evaluaron la capacidad funcional del paciente mediante el índice Karfnosky. Ambos cuestionarios estaban codificados con un mismo número que permitía la posterior relación durante el análisis, garantizando el anonimato del paciente.

Se utilizó el índice de Karfnosky ${ }^{13}$ para medir el grado de autonomía, que utiliza una escala de diez niveles con puntuaciones que van de 0 (muerte) a 100 (autonomía total).

El instrumento de medida utilizado para la valoración de la satisfacción del paciente fue una modificación del cuestionario SERVQHOS ${ }^{14}$, del que se eliminó una pregunta que carecía de interés en estos pacientes y se incluyeron dos preguntas relativas a la dieta y a la medicación.

E I cuestionario resultante consta de tres partes diferenciadas. La primera con 20 ítems de fácil comprensión y respuesta; abarca los elementos básicos en la evaluación de la calidad percibida, incluyendo la calidad de la atención sanitaria. EI paciente puntúa cada una de estas preguntas en una escala de Likert, que va desde 1: la asistencia ha sido mucho peor de lo que esperaba a 5: mucho mejor de lo que esperaba. E $n$ la segunda parte se pregunta sobre la satisfacción global respecto a la unidad y al hospital, mientras que la tercera parte recoge características sociodemográficas de los pacientes (Anexo 1).

Análisis. Para el análisis de datos se utilizó el programa SPSS 12.0 para Windows, donde se exportó toda la información desde una base de datos creada al efecto, mediante la que se obtuvieron las frecuencias y medias de puntuación en cada ítem. Se utilizó el Alpha de Cronbach para el análisis de fiabilidad de los datos. Se calculó la media global de satisfacción y a partir del análisis de regresión de los datos se determinaron las variables con más peso en la satisfacción de los pacientes.

\section{Resultados}

De los 81 cuestionarios entregados (puesto que tres pacientes fallecieron antes de cumplimentarlo) se re- 
cogieron 77 (tasa de respuesta 95,06\%). De los pacientes encuestados $55(71,4 \%)$ recibían tratamiento con hemodiálisis (HD) y $22(28,6 \%)$ con diálisis peritoneal (DP).

De los 55 pacientes en HD el $65 \%$ eran hombres y el $35 \%$ mujeres, con una edad media de $68,8 \pm 14,06$ años y un tiempo medio en HD de 44,9 $\pm 52,2$ meses. De los 22 pacientes en DP el $82 \%$ eran hombres y el $18 \%$ mujeres. La edad media era de $54,6 \pm 12,4$ años, con un tiempo medio en DP de $14,04 \pm 10,85$ meses.

Se utilizó el Alpha de Cronbach para el análisis de fiabilidad y validez de los datos, obteniendo un valor de 0,95 en los cuestionarios de HD, y de 0,90 en los de DP, (superior a 0,7 que indica que estos son fiables).

Del análisis de medias de las dimensiones exploradas en la primera parte del cuestionario observamos que los pacientes en HD puntúan todos los ítems por encima de 3,5, excepto en la facilidad para llegar al hospital. En los pacientes en DP podemos apreciar una puntuación superior a 4 en todas las dimensiones excepto en el tiempo de espera para ser atendido por el médico. El ítem que obtiene una mayor puntuación en los pacientes en HD es si el personal cumple lo que dice que va a hacer y en DP el trato personalizado que se da a los pacientes (tabla1).

\begin{tabular}{|c|c|c|c|c|c|c|c|c|c|}
\hline & & \multirow{2}{*}{$\begin{array}{l}\mathbf{N} \\
44\end{array}$} & \multicolumn{5}{|c|}{ \% Puntuación Escala de Likert } & \multirow[t]{2}{*}{ Media } & \multirow{2}{*}{$\begin{array}{l}\text { Desv. } \\
\text { Típ. }\end{array}$} \\
\hline & & & 1 & 2 & 3 & 4 & 5 & & \\
\hline Tecnología de los equipos & DP & 21 & 0 & 0 & 19 & 28,6 & 52,4 & 4,33 & 0,795 \\
\hline \multirow[t]{2}{*}{ Apariencia del personal } & HD & 53 & 0 & 5,7 & 28,3 & 17 & 49 & 4,09 & 1 \\
\hline & DP & 22 & 0 & 4,5 & 13,6 & 18,2 & 63,7 & 4,40 & 0,908 \\
\hline \multirow[t]{2}{*}{ EI personal cumple lo que dice } & HD & 52 & 2 & 2 & 25 & 25 & 46 & 4,11 & 0,983 \\
\hline & DP & 22 & 0 & 0 & 9,1 & 18,2 & 72,7 & 4,63 & 0,657 \\
\hline \multirow[t]{2}{*}{ E stado de las salas } & HD & 52 & 2 & 0 & 38,4 & 21,1 & 38,5 & 3,94 & 0,978 \\
\hline & DP & 22 & 0 & 9,1 & 18,2 & 22,7 & 50 & 4,13 & 1,037 \\
\hline \multirow[t]{2}{*}{ Información médica } & HD & 53 & 1,9 & 11,4 & 22,6 & 20,7 & 43,4 & 3,92 & 1,141 \\
\hline & DP & 21 & 0 & 0 & 4,7 & 23,8 & 71,5 & 4,66 & 0577 \\
\hline \multirow[t]{2}{*}{ Tiempo de espera consulta medica } & HD & 50 & 4 & 8 & 40 & 14 & 34 & 3,66 & 1,153 \\
\hline & DP & 22 & 0 & 4,5 & 31,8 & 27,3 & 36,4 & 3,95 & 0,950 \\
\hline Facilidad para llegar al hospital & HD & 51 & 7,8 & 9,8 & 33,4 & 23,5 & 25,5 & 3,49 & 1,206 \\
\hline \multirow[t]{2}{*}{ Interés de E nfa solventar problemas } & HD & 53 & 5,6 & 5,7 & 30,2 & 28,3 & 30,2 & 3,71 & 1,133 \\
\hline & DP & 22 & 0 & 0 & 4,5 & 27,3 & 68,2 & 4,63 & 0,581 \\
\hline \multirow[t]{2}{*}{ Puntualidad sesión de HD } & HD & 53 & 0 & 15 & 35,9 & 28,3 & 20,8 & 3,54 & 0,991 \\
\hline & DP & 21 & 0 & 0 & 14,3 & 9,5 & 76,2 & 4,61 & 0,740 \\
\hline \multirow[t]{2}{*}{ Rapidez conseguir lo que necesita } & HD & 51 & 5,9 & 11,7 & 29,4 & 29,4 & 23,6 & 3,52 & 1,155 \\
\hline & DP & 22 & 0 & 0 & 9,1 & 27,3 & 63,6 & 4,54 & 0,670 \\
\hline \multirow[t]{2}{*}{ Disposición del personal para ayudar } & HD & 53 & 7,5 & 7,5 & 22,7 & 24,5 & 37,8 & 3,77 & 1,250 \\
\hline & DP & 22 & 0 & 0 & 4,5 & 9,1 & 86,4 & 4,81 & 0,501 \\
\hline \multirow[t]{2}{*}{ Confianza que el personal transmite } & HD & 52 & 5,8 & 3,8 & 25 & 32,7 & 32,7 & 3,82 & 1,115 \\
\hline & DP & 22 & 0 & 0 & 0 & 18,2 & 81,8 & 4,81 & 0,394 \\
\hline \multirow[t]{2}{*}{ Amabilidad del personal } & HD & 53 & 9,4 & 3,8 & 28,3 & 28,3 & 30,2 & 3,66 & 1,223 \\
\hline & DP & 22 & 0 & 0 & 4,5 & 4,6 & 90,9 & 4,86 & 0,467 \\
\hline \multirow[t]{2}{*}{ Preparación del personal } & HD & 53 & 1,9 & 1,9 & 35,8 & 28,3 & 32,1 & 3,86 & 0,961 \\
\hline & DP & 21 & 0 & 0 & 10,5 & 15,8 & 73,7 & 4,19 & 1,53 \\
\hline \multirow[t]{2}{*}{ Trato personalizado } & HD & 52 & 7,7 & 3,8 & 34,6 & 17,3 & 36,6 & 3,71 & 1,133 \\
\hline & DP & 21 & 0 & 0 & 4,8 & 4,8 & 90,4 & 4,85 & 0,478 \\
\hline \multirow[t]{2}{*}{ Interés de E nfa por los pacientes } & HD & 52 & 3,8 & 5,8 & 28,8 & 23,1 & 38,5 & 3,86 & 1,120 \\
\hline & DP & 22 & 0 & 0 & 9,5 & 19 & 71,5 & 4,4 & 1,181 \\
\hline \multirow[t]{2}{*}{ Comunicación con familiares } & HD & 50 & 8 & 8 & 28 & 26 & 30 & 3,62 & 1,227 \\
\hline & DP & 21 & 0 & 0 & 14,3 & 23,8 & 61,9 & 4,47 & 0,749 \\
\hline \multirow[t]{2}{*}{ Información referente a medicación } & HD & 51 & 5,9 & 1,9 & 29,4 & 27,5 & 35,3 & 3,84 & 1,120 \\
\hline & DP & 21 & 0 & 0 & 19 & 9,5 & 71,5 & 4,52 & 0,813 \\
\hline Información referente a dieta & HD & 52 & 5,8 & 7,7 & 34,6 & 19,2 & 32,7 & 3,65 & 1,186 \\
\hline & DP & 22 & 0 & 0 & 22,7 & 18,2 & 59,1 & 4,36 & 0,847 \\
\hline
\end{tabular}

Tabla 1: Puntuación media de los atributos de satisfacción del SERVQHOS. 
Finalmente se realizó un análisis factorial agrupando las distintas variables, a partir del cuál se hizo un análisis de regresión, determinando que los factores con más peso en la satisfacción de los pacientes en HD eran si el personal cumple lo que dice que va a hacer, el trato personalizado que se da, la capacidad del personal para comprender las necesidades de los pacientes, la confianza que transmite el personal y la información proporcionada por el médico; sin embargo, en DP, éstos eran la comunicación que el personal de la unidad mantiene con los familiares y la información sobre la medicación.

La satisfacción media global calculada (escala de 1 a 4) de nuestra población fue de 3,28 en los pacientes en HD y de 3,68 en los pacientes en DP.

En la figura 1 se puede apreciar el nivel de satisfacción de los pacientes en HD y DP con los cuidados sanitarios recibidos durante su estancia en la U nidad de Diálisis, (aspecto recogido en la segunda parte del cuestionario).

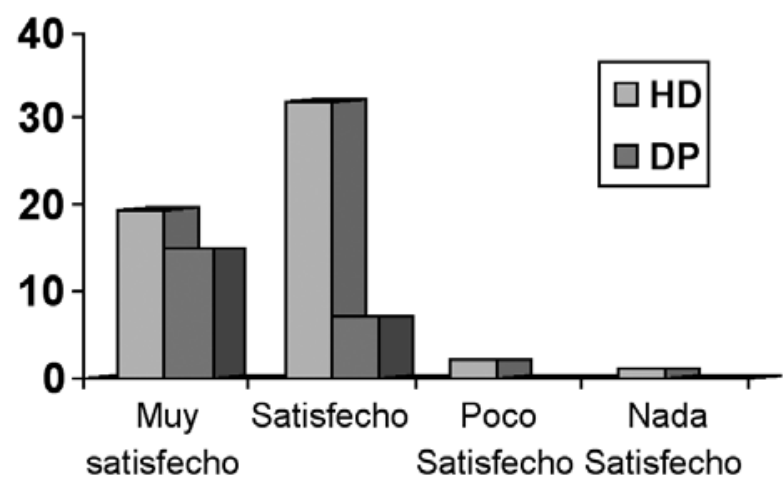

Figura 1. Nivel de satisfacción de los pacientes.

E $n$ relación con las variables sociodemográficas de los pacientes (edad, sexo, estado civil, nivel de estudios y situación laboral) no encontramos correlación con el nivel de satisfacción. La media de puntuaciones del índice de Karnofsky en los pacientes en HD es de 65-69, inferior a las obtenidas en DP (con valores de 76-78). No encontramos correlación entre el índice de Karfnosky y la satisfacción media global en pacientes en HD o DP (figura 2).

\section{KARNOFSKY - SATISFACCION GLOBAL}

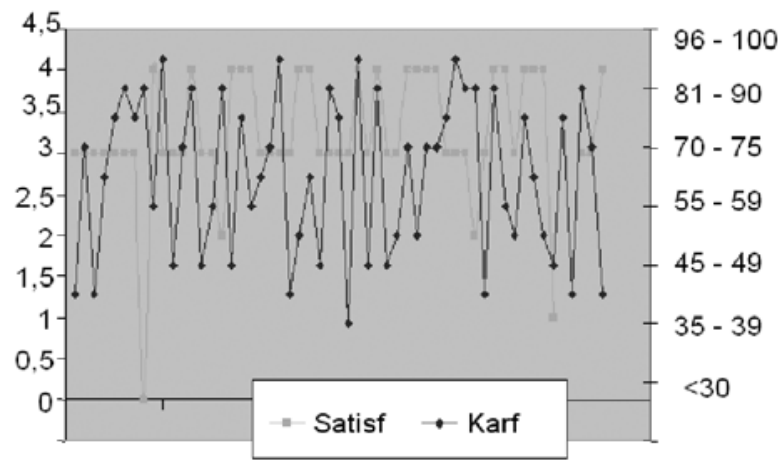

Figura 2.

\section{Discusión}

La valoración del nivel de satisfacción de los pacientes es un instrumento que utilizado periódicamente ofrece la oportunidad de monitorizar atributos de la calidad percibida como: tangibilidad, fiabilidad, seguridad, empatía y capacidad de respuesta7. La satisfacción con cualquier servicio, como la atención sanitaria, se consigue cuando el usuario percibe que el servicio prestado supera sus expectativas ${ }^{15}$. Podemos observar que los pacientes en DP presentan un nivel de satisfacción mayor que los pacientes en HD, aunque son muchos y variados los aspectos que deben abordarse al referirnos al paciente renal.

E $\mathrm{n}$ los últimos años se está produciendo un aumento en el número de pacientes en DP, hecho que parece relacionarse con la participación del paciente en la elección del tipo de tratamiento renal sustitutivo ${ }^{16,17}$ y que viene apoyado por la L ey 41/2002 de 14 de noviembre reguladora de la Autonomía de los Pacientes, que en su artículo 8 dice: "Toda información en el ámbito de salud de un paciente necesita el consentimiento libre y voluntario del afectado una vez que, recibida la información prevista en el artículo 4, haya valorado las opciones propias del caso". Podemos observar que el $100 \%$ de los pacientes en DP había pasado por la consulta de enfermería prediálisis, frente un $60 \%$ de los pacientes en HD.

La diálisis peritoneal es un tratamiento domiciliario, en el que el propio paciente o un familiar asumen la 
realización de la técnica; para lo que es necesario un periodo de entrenamiento. Al estudiar las variables con más peso en la satisfacción de los pacientes, podemos apreciar que en la DP son la comunicación que el personal de la unidad mantiene con los familiares y la información sobre la medicación, aspectos relacionados con la responsabilidad que tienen que asumir al realizar la técnica. Sin embargo en HD los aspectos determinantes de la satisfacción son si el personal cumple lo que dice que va a hacer, el trato personalizado que se da; la capacidad del personal para comprender las necesidades de los pacientes, la confianza que transmite el personal y la información proporcionada por el médico; estando estos aspectos de la asistencia directamente relacionados con la atención que reciben; las intervenciones sobre estos atributos permitirán mejorar la satisfacción de los pacientes.

Las características sociodemográficas no parecen tener gran relevancia en el nivel de satisfacción de los pacientes, hecho que también ha sido observado por otros autores ${ }^{18}$.

La capacidad funcional de los pacientes en DP es superior a la de los pacientes en HD, aspecto este observado por otros autores en el momento de iniciar tratamiento renal sustitutivo ${ }^{11}$. Son varias las publicaciones que relacionan la capacidad funcional con la edad media de los pacientes ${ }^{19,20}$. E n nuestro estudio observamos como los pacientes en DP presentan una edad media inferior a los pacientes en HD y una puntuación superior en índice Karfnosky, aunque no debemos olvidar que existen otros factores que pueden influir en este aspecto, y tal vez sean los pacientes que se encuentran mejor los que deciden realizar el tratamiento renal sustitutivo de forma autónoma ${ }^{21}$.

\section{Conclusiones}

E I nivel de satisfacción de los pacientes en DP es mayor que el de los pacientes en HD en la población objeto de estudio. No encontramos correlación entre la capacidad funcional y la satisfacción media global de los pacientes en HD o DP.

Los pacientes en hemodiálisis presentan unas puntuaciones inferiores en el índice de Karfnosky a los pacientes en DP. Las variables que determinan el grado de satisfacción de los pacientes en HD son diferentes a las de los pacientes en DP.

\section{Agradecimientos}

Agradecemos la colaboración de todos los pacientes y nuestros compañeros, sin los cuales este trabajo no hubiera sido posible.

\section{Bibliografía}

1. J anse AJ, Uiterwaal CS, Gemke RJ, Kimpen J L, Sinnema G. A difference in perception of quality of life in chronically ill children was found between parents and pediatricians. J Clin Epidemiol. 2005; 58:495-502.

2. Svavarsdottir EK, Rayens MK. Hardiness in families of young children with asthma. J Adv Nurs. 2005; 50:381-90.

3. Diaz Rodriguez C, Rodriguez-Arias Palomo J L, Canosa Diz J , Venero Celis M, Agra Tuna S, Otero L arrea M, Ribeiro Pazos S, Durana Tonder B, Prado Meis M del C, Varela N. Preliminary report on multiple family discussion groups for patients with chronic medical illness and its repercussions in the management of the hemodialysis process. Ther Apher Dial. 2004; 8:492-6.

4. Raina C, Kerse N, Arroll B, RobinsonE . E ffectiveness of counselling patients on physical activity in general practice: cluster randomised controlled trial. BMJ . 2003; 326793.

5. B ritten N. Patients' expectations of consultations. B MJ . 2004; 328: 416-417.

6. Hernández Conesa J. Historia de la Enfermería. Interamericana McGraw-Hill, Madrid,1995.

7. Mira J J , B uil J A, Aranaz J , Vitaller J , L orenzo S, I gnacio E, R odriguez-M arin J , A guado H, Gimenez A. ¿Qué opinan los pacientes de los hospitales públicos? Análisis comparativo de los niveles de calidad percibida en cinco hospitales. Gac Sanit. 2000; 14:291-3.

8. Arenas MD, Moreno E, Reig A, Millan I, E gea JJ , A moedo M L, Gil M T, Sirvent AE . E valuación de la calidad de vida relacionada con la salud mediante las láminas Coop-Wonca en una población de hemodiálisis. N efrología. 2004; 24:470-9. 
9. Comité de Registro de la SEN: Amenábar JJ, García-López F, Robles NR, Saracho R. Informe de Diálisis y Trasplante de la Sociedad E spañola de Nefrología y Registros Autonómicos correspondiente al año 1999. Disponible en: http:/www. senefro.org/grupost.htm. (consultado el 27 de abril de 2005)

10. U.S.Renal Data System. USRDS 2000 Annual Data Report: atlas of end-stage renal disease in the United States. National Institutes of Health, National Institute of Diabetes and Digestive and Kidney Disease, B ethesda, M D. 2000.

11. Gutierrez Vilaplana JM, Pitarch del Amor G, I bars i Moncasi P. Calidad de Vida y decisión de técnica de tratamiento renal sustitutivo. Libro de comunicaciones del XXIX Congreso Nacional de la Sociedad E spañola de E nfermería Nefrológica (SE DE N) Octubre 2004.

12. López K, García FJ, De Álvaro F. Salud percibida, estado funcional y mortalidad en pacientes diabéticos en tratamiento renal sustitutivo: diseño del estudio CALVIDIA. Nefrología. 1997; XVII:296-203.

13. Karnofsky DA, B urchenal J MK. The clinical evaluation of the therapeutic agent. $\mathrm{N}$ York Columbia U niversity Press 1949.

14. Mira JJ, Aranaz J, Rodríguez-Marín J, Buil J A, Castell M, Vialler J. SERVQHOS: un cuestionario para evaluar la calidad percibida de la asistencia hospitalaria. M edicina P reventiva. 1998; IV: 12-8.
15. Parasuraman A, Zeithaml V, Berry L. SERVQUAL: a multiple-item scale for measuring consumer perceptions of Service Quality. J Retailing 1988; 65:2-40.

16 .Parmar MS. Chronic renal disease BMJ 2002; 325:85-90.

17. http://www.kidneyschool.org/ (consultado el 27 de abril de 2005)

18. Hall J A, Dornan MC. Patient sociodemographic characteristics as predictors of satisfaction with medical care: a meta-analysis. Soc Sci Med 1990; 30:811-818.

19. Martín A, García A, Huebra D, Rodríguez 0 , Chico M. Tolerancia a la diálisis de los pacientes mayores de 75 años. Libro de comunicaciones del XXIII Congreso Nacional de la Sociedad española de E nfermería Nefrológica. Sevilla. 1998: 34-42.

20. M oreno E, Arenas M D, Castell G, Cantó MJ , E scalant $L$, Porta $E$, et al. $E$ studio de la calidad de vida relacionada con la salud en una población de hemodiálisis mediante las láminas de COOP/WONCA. Libro de comunicaciones del XXVIII Congreso Nacional de la Sociedad española de E nfermería Nefrológica. Palma de Mallorca. 2003:253-256.

21. García R, Gallardo P, Rojas A, Tejuca A, Tejuca M : Grado de satisfacción de pacientes con IRC en diálisis hospitalaria vs diálisis domiciliaria. Libro de comunicaciones del XXI Congreso Nacional de la Sociedad E spañola de E nfermería N efrológica. Salamanca. 


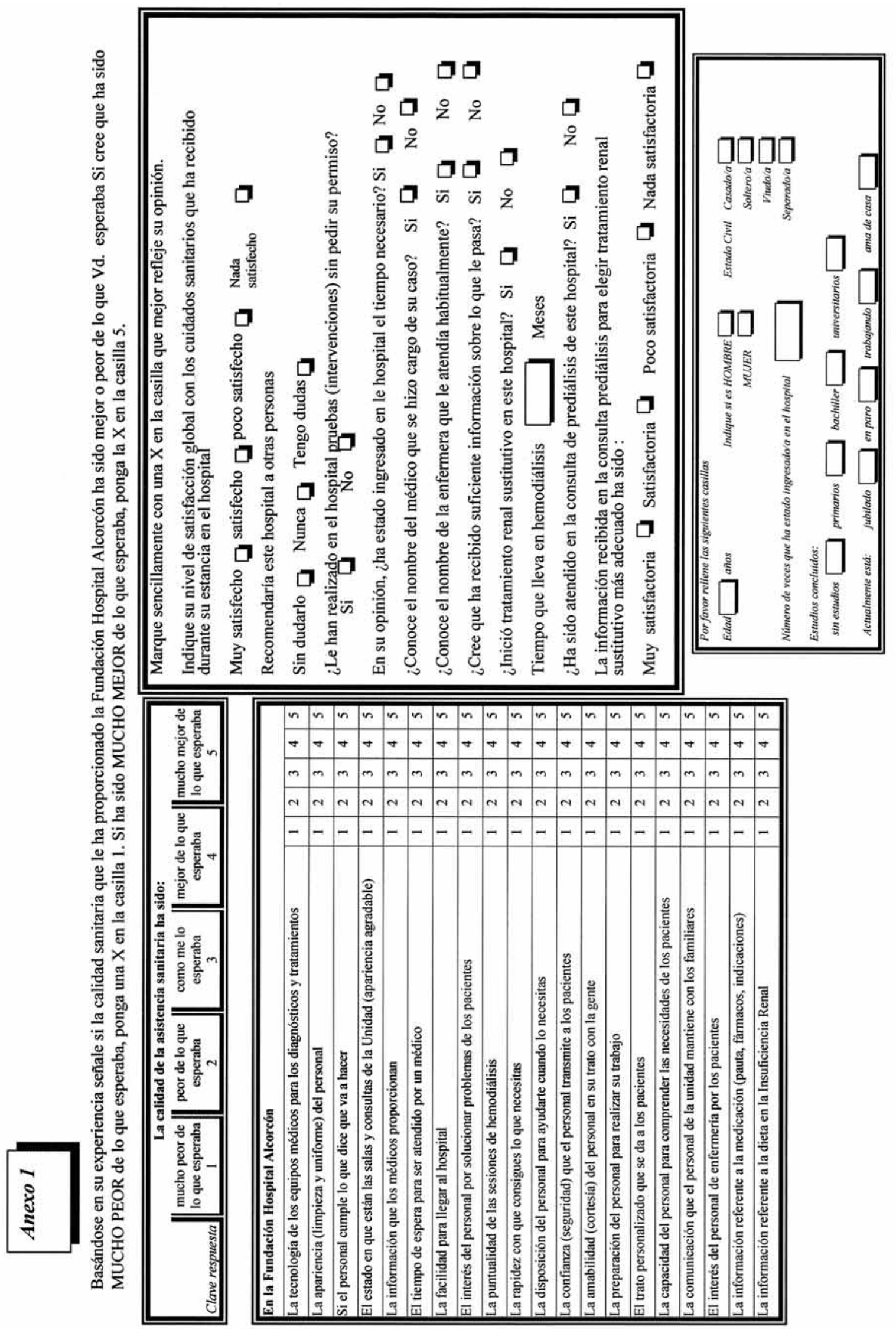

